\title{
Die Dimensionen des Reiseberichts
}

Verschaffen wir uns zunächst einmal einen Überblick über die unterschiedlichen Dimensionen des Reiseberichts im Raum und in der Zeit sowie in seiner Verankerung hinsichtlich anderer, noch genauer zu erläuternder Parameter. Dabei wählen wir nun literarische und andere Beispiele aus, die vorwiegend nicht mehr aus der Zeit der ersten Phase beschleunigter Globalisierung stammen, sondern der zweiten Phase beschleunigter Globalisierung entnommen sind und damit jenem Zeitraum angehören, der sich in der zweiten Hälfte des 18. Jahrhunderts situiert und nicht mehr vorrangig von den iberischen Mächten, sondern von den neuen europäischen Expansionsmächten England und Frankreich beherrscht wird. Dadurch soll uns die Vorlesung geradezu nebenbei einen Einblick in jene Beschleunigungsphase vermitteln, in der nicht mehr die Karavelle, sondern die Fregatte das globalisierende Verkehrsmittel ist und in der die Globalisierungsängste nicht mehr vorrangig von der Syphilis, sondern vom Gelbfieber ausgelöst wurden. Es ist eine Globalisierungsphase, die sich überdies genau in jenem Zeitraum ansiedelt, der für uns in dieser Vorlesung das take-off der Moderne darstellt.

In Rückgriff auf eine Bemerkung des französischen Anthropologen und Strukturalisten Claude Lévi-Strauss, der in seinem berühmt gewordenen Band Tristes Tropiques betont hatte, dass sich Reisen in zumindest fünf Dimensionen ansiedeln, ${ }^{1}$ gilt es zunächst festzuhalten, dass die beiden ersten Dimensionen des Raumes gerade in der kartographischen Erfassung und Auswertung der untersuchten Reisen anschaulich werden. Denn die Kartographie ist die Kunst, Räume auf eine plane Fläche zu projizieren und anschaulich zu machen. Die Reisende oder der Reisende bewegen sich gleichsam innerhalb eines zweidimensionalen Koordinatensystems entlang einer Linie, die sich vor allem in den ersten handschriftlichen Aufzeichnungen und darauf beruhenden ersten kartographischen Ausarbeitungen mit aller wünschenswerten Deutlichkeit niederschlägt. Damit werden Bewegungen des Reisenden innerhalb eines zweidimensionalen Raumes vor Augen geführt, der in seiner Darstellung selbst schon eine Abstraktion darstellt.

Beschäftigen wir uns also zunächst mit den beiden ersten Dimensionen des Raumes. Der wohl bekannteste deutschsprachige Reisende des ausgehenden 18. und des 19. Jahrhunderts und der sicherlich berühmteste Lateinamerikaforscher seiner Zeit, Alexander von Humboldt, hat in seine Amerikanischen Reisetagebücher kartographische Aufnahmen der von ihm befahrenen Flüsse eingezeichnet, welche die Bewegungen des Reisenden etwa auf dem Orinoco und Casiquiare, aber auch später auf dem Río Magdalena zeigen. Es handelt

1 Vgl. Lévi-Strauss, Claude: Tristes Tropiques. Paris: Plon 1955.

Ә Open Access. (c) 2020 Ottmar Ette, publiziert von De Gruyter. (cc))BY-NC-ND Dieses Werk ist lizenziert unter der Creative Commons Attribution-NonCommericial-NoDerivatives 4.0 Lizenz.

https://doi.org/10.1515/9783110650686-006 
sich dabei um erste Zeichnungen mit genauen Eintragungen, aus denen im Nachgang erstaunlich präzise Karten wurden. Diese Zeichnungen zeigen das linienhafte Vordringen des Reisenden entlang einer zweidimensionalen Linie oder Achse: sie situieren den Reisenden und seine Bewegungen in den ersten beiden Dimensionen des Raumes, ohne ihn freilich in der Höhe zu kartographieren. Dies ist gewiss auch nicht notwendig, da sich die Reisebewegungen auf einen Fluss konzentrieren, der bekanntlich einem mehr oder minder sanft gerichteten Abfallen folgt.

Alexander von Humboldts Zeichnungen vom Río Magdalena im heutigen Kolumbien etwa beschränken sich auf eine vielfach gewundene Linie, die wir genau verfolgen können, sowie einen scharf begrenzten schmalen, Saum von Gebirgsschraffen, welche die vom Fluss aus sichtbaren Höhenzüge andeuten sollen. All dies charakterisiert einen Bereich des Sehens, gleichsam eine Sichtachse, welchen der preußische Naturforscher aus eigener Anschauung erfassen und in sein Reisetagebuch eintragen konnte. ${ }^{2}$

Dabei ergänzen schriftliche Notizen die visuellen Zeichen, die belegen, wie eng der Gesichtskreis und wie tunnelartig die Perspektive blieben, die sich dem Reisenden vom Fluss aus bieten musste. Mehr war weder zu erblicken noch in die jeweilige Karte einzutragen. Diese Flusszeichnung wurde möglichst präzise ausgeführt, bildete sie doch die Grundlage für weitere, sich anschließende Bearbeitungen und Ausarbeitungen einer möglichst genauen Kartographie der gesamten Flussregion. doch noch sind wir weit von einem wirklichen Kartenbild entfernt.

Betrachten wir aber dann die Ausarbeitung einer topographischen Karte, sowie sie Humboldt in seinen Kartenwerken vorlegte. Die grundlegende Einzeichnung des Flussverlaufes ist in ihr noch immer nachvollziehbar; doch Entscheidendes in der graphischen Darstellung hat sich geändert. Eine abgeschlossene topographische Karte überspielt eine solche tunnelartige Perspektive langsamen Abtastens einer gewundenen Linie, inszeniert sie doch stets einen alles umgreifenden Blick von oben, ein von Humboldt stets aufgerufenes und geschätztes „Schweben über den Dingen“3 aus einem Blickwinkel,

2 Vgl. die Reproduktion seiner Karten von der Kolumbienreise in Humboldt, Alexander von: In Kolumbien. En Colombia. Auswahl aus seinen Tagebüchern, herausgegeben von der Akademie der Wissenschaften der Deutschen Demokratischen Republik und der Kolumbianischen Akademie der Wissenschaften. Bogotá: Publicismo y Ediciones 1982, S. 29a-34a.

3 Diese Formulierung stammt aus einem Brief Alexander von Humboldts vom 28. April 1841 an Varnhagen von Ense, wo es in Bezug auf seinen Kosmos heißt: „Der eigentliche Zweck ist das Schweben über den Dingen, die wir 1841 wissen. “ Briefe von Alexander von Humboldt an Varnhagen von Ense aus den Jahren 1827 bis 1858. Herausgegeben von Ludmilla Assing. Leipzig: Brockhaus 1860, S. 92. 


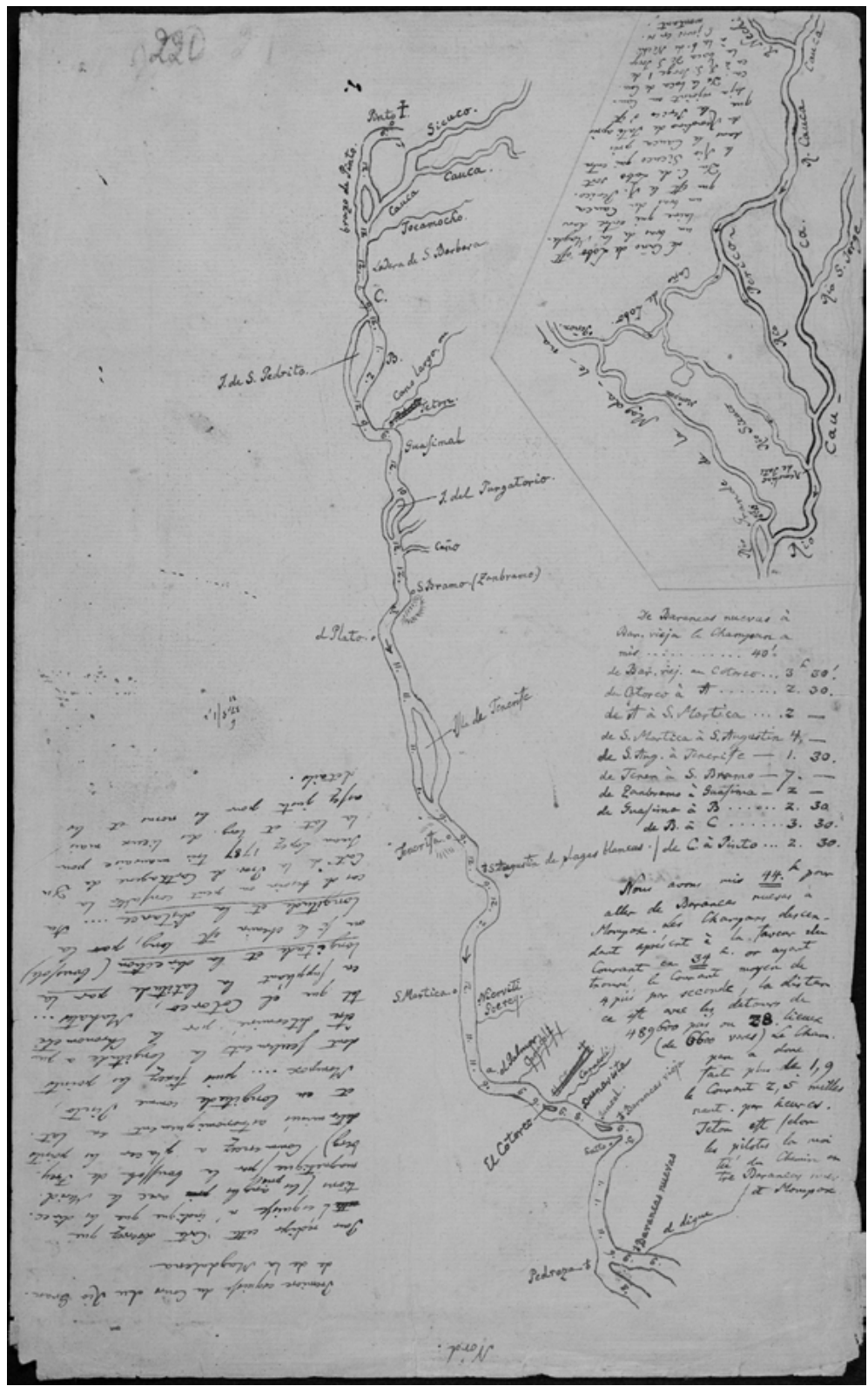

Abb. 22: Alexander von Humboldts Skizze des Río Magdalena aus seinem Reisetagebuch VIla/b, „Erster Entwurf des Verlaufs des Río Grande de la Magdalena“, 1801. 
der nicht mehr der eines konkreten Subjekts und seines beschränkten Gesichtskreises ist. Eine topographische Karte versucht, jenseits des individuellen Blickpunktes eine allgemeine, überindividuelle Position so einzunehmen, dass daraus gleichsam ein allwissender Blick auf eine Region entsteht, die kartographiert werden soll.

Die Erstellung einer topographischen Karte ist daher gleichbedeutend mit einer Überführung linienhafter individueller Erfahrung über verschiedene Zwischenstadien in eine flächenhaft ausgebreitete Überschau, die auf einem (Karten-)Netz beruht, das eine vom Reisenden allein nie zu erzeugende Vollständigkeit suggeriert. Die jeweiligen Quellen für diese suggerierte Vollständigkeit bleiben dabei in der Regel obskur oder diffus. Noch immer bewegen wir uns freilich auch auf der Ebene einer topographischen Karte auf der Ebene zweier Dimensionen, in welche die Höhe eher symbolisch eingetragen und durch Gebirgsschraffen angedeutet wird.

Zugleich muss eine derartige Karte doch stets ausschnitthaft bleiben und gibt gerade dadurch einen (diegetischen) Rahmen vor, innerhalb dessen sich Raum, Zeit und Handlung des eigentlichen Reiseberichtes situieren lassen. Die Amerikanischen Reisetagebücher des preußischen Naturforschers und Schriftstellers bieten das faszinierende Schauspiel, wie sich Rahmen und Inhalt seines so oft beschworenen 'Naturgemäldes', wie sich Linie und Fläche gegenseitig hervorbringen. So wird aus einer Flussfahrt, die sich innerhalb eines eng begrenzten Sichtfeldes vollzog, die Erzeugung einer Fläche, die sich im Kartenbild nicht als Fiktion zu erkennen gibt, wohl aber auf vielen Mutmaßungen sowie zusätzlich - etwa aus früheren Kartenwerken eingeholten Informationen beruht. Wir wohnen der Entstehung einer kartographisch erzeugten Fläche bei.

Die Konstruktionsprinzipien einer solchen Karte zeigen sich nicht zuletzt in der Materialität seiner Einträge in das Kartenbild. Schon in der ersten Flusszeichnung werden diese Elemente klar erkennbar. Denn den in Humboldts Tagebüchern auf dem Papier freibleibenden Raum füllen - nicht nur aus Gründen eines gezwungenermaßen haushälterischen Umgangs mit dem kostbaren Material - ausführliche schriftliche Zusätze, welche sich der Form des Flussverlaufs anpassen und die leergebliebenen großen Flächen ausfüllen. Bild und Text stehen nicht nur in einem wechselseitigen Illustrationsverhältnis: Sie durchdringen sich und erzeugen eine Karte, in der sich Bild und Schrift wechselseitig queren und durchdringen.

Man darf hierin nicht allein den Ausdruck jenes horror vacui erkennen, der die unbekannten Gebiete frühneuzeitlicher Karten mit allerlei Ungeheuern und Fabelwesen schmückte und jene Leerflächen zum Verschwinden brachte, in die es keine Informationen einzutragen gab. Dem Ineinanderwirken von 


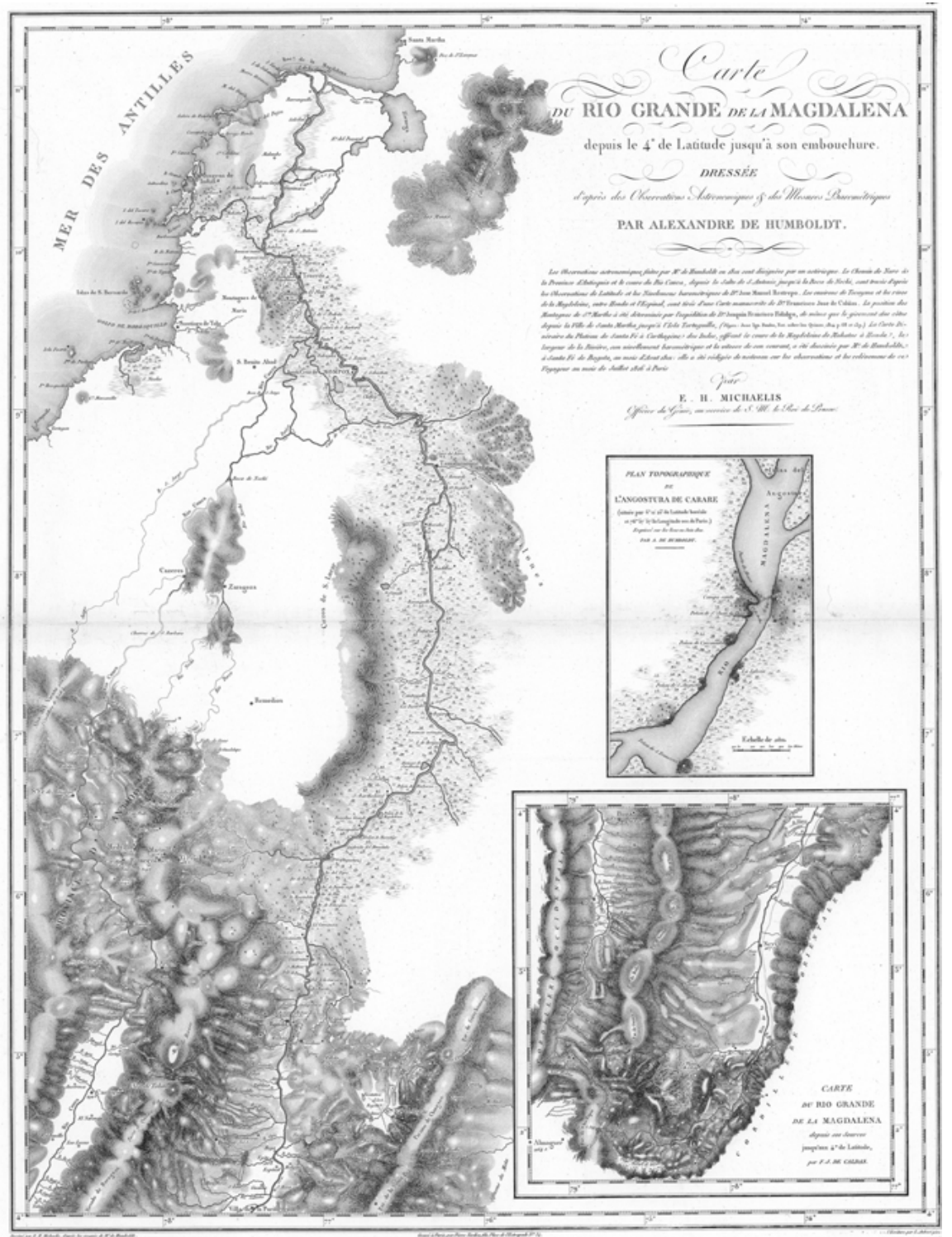

Abb. 23: Karte des Rio Grande de la Magdalena aus dem Atlas géographique et physique des régions équinoxiales du Nouveau Continent, Alexander von Humboldt, 1834. 
Bild und Schrifttext ist vielmehr ein epistemologischer Status zuzuerkennen, insoweit der vom Auge erfasste Bereich erweitert wird durch jene Informationen, die der Forscher während seiner Reise von anderen Informanten bezog und eigenhändig zusammentrug. Bild und Schrift, Handschrift und Schriftbild durchdringen sich wechselseitig und erzeugen eine Fläche, die der reisende selbst lediglich $\mathrm{zu}$ durchqueren vermochte. Freilich konstituiert sich diese Fläche durch jene Bewegungen, die sie queren und durchqueren.

Doch es gibt noch etwas Anderes, das zweifellos von nicht geringerer epistemologischer Relevanz ist. Denn das Gesehene verbindet sich mit dem Gehörten und Gelesenen, das Nicht-Gewusste mit dem Vor-Gewussten beziehungsweise mit zugänglichen Wissensbeständen, Auge und $\mathrm{Ohr}^{4}$ verknüpfen sich hier miteinander, um die Leere des Unbekannten aus dem definitiven Kartenbild - wenn auch keineswegs immer vollständig - zu verdrängen und mit Informationen unterschiedlichster Provenienz auszufüllen. In einem Tagebucheintrag hat der preußische Gelehrte gegenüber ersten skeptischen und ablehnenden Reaktionen von Seiten kolonialspanischer Behörden selbst auf die Prozesshaftigkeit seiner kartographischen Arbeit hingewiesen, ohne es freilich am notwendigen Selbstbewusstsein fehlen zu lassen. So kommentierte er zu seiner Karte:

Die Einzelheiten sind sehr zutreffend, die kleinsten laderas finden sich verzeichnet, es handelt sich um die erste Karte (Plan), die jemals von diesem Fluß aufgenommen wurde, allen Ingenieuren zum Trotz, die ihn während der vergangenen 300 Jahre hinauffuhren. Ich habe das Unglück, ein Ausländer zu sein [...]. Für wie exakt ich meine Arbeit mit guten Gründen auch immer halten mag, so wird sie doch immer für schlecht gehalten werden, da sie von einem Preußen stammt. Im übrigen ist meine Karte ein erster Versuch, und ich zweifle nicht daran, daß man sie noch berichtigen könnte. ${ }^{5}$

Es ist fürwahr ein gewaltiger Sprung bis zur Fertigstellung seines topographischen Kartenwerkes. Noch in der großartigen Doppelseite von Humboldts Atlas géographique et physique du Nouveau Continent, die den Magdalenenstrom beziehungsweise einen Ausschnitt aus dem heutigen Kolumbien zeigt, ${ }^{6}$ sind Flächen ohne Eintragungen vorhanden. Doch füllen hier kartographische Detailaufnahmen einzelner Flussabschnitte geschickt die Lücken, die beim besten Willen nicht informationsreich auszufüllen waren. Der Bereich des Wissens ist bedeutend ausgeweitet, weit über das vom Auge des einzelnen

4 Vgl. hierzu Teil III dieses Bandes.

5 Humboldt, In Kolumbien, S. 31.

6 Ebda., o.S. Diese Karte ist leicht zugänglich in Hein, Wolfgang-Hagen (Hg.): Alexander von Humboldt. Leben und Werk. Frankfurt am Main: Weisbecker Verlag 1985, S. 244. 
Reisenden Erfassbare hinaus. Der Übergang vom Reisetagebuch zum Reisebericht verläuft parallel zu dieser Entwicklung, wenn auch gemäß der eigenen Regeln dieses literarischen Genres. Die Amerikanischen Reisetagebücher demonstrieren diesen Transformationsprozess und führen uns vor Augen, wie viele Informationen noch einfließen mussten, bis aus dem Reisetagebuch ein literarischer Reisebericht und bis aus der Flusszeichnung eine kartographische Karte entstehen konnten. Und doch: wir haben die Zweidimensionalität des Raumes noch nicht verlassen.

Die dritte Dimension des Raumes ist jene, die gerade der Reisebericht des ausgehenden 18. und beginnenden 19. Jahrhunderts sich zur Aufgabe macht und erforscht. Es erscheint überraschend, aber erst während dieses Zeitraumes können wir eine Betonung dieser Dimension erkennen und zugleich auch eine Ästhetisierung dieser dritten Dimension der Höhe (oder der Tiefe) beobachten. So gibt es kaum einen Reisebericht in jenem Zeitraum, in welchem sich nicht auch eine Bergbesteigung findet.

Mehr noch: Der Blick von oben entwirft ebenso eine Theorie der Landschaft wie eine Landschaft der Theorie, wobei der Transparenz dieses Blickes eine zugleich literarische und epistemologische Bedeutung zukommt. Unter einer Theorie der Landschaft mit ihren jeweiligen Elementen kann man sich vielleicht noch etwas vorstellen. Aber was in aller Welt ist eine Landschaft der Theorie?

Sagen wir es zunächst allgemein: Literatur und Wissenschaft, Theorie und Praxis verbinden sich in derartigen Landschaften der Theorie oftmals aufs engste. Eine Landschaft der Theorie kann uns - kurz gesagt - Auskunft darüber geben, wie die Theorie der Reise beziehungsweise des Reiseberichts aussieht und welche Schwerpunkte sie setzt. Sie ist, allgemein gesprochen, eine Veranschaulichung, eine Visualisierung jener Theorie und Epistemologie, die einem Reisebericht zugrunde liegen.

Nehmen wir ein Beispiel wiederum aus der zweiten Phase beschleunigter Globalisierung. Exemplarisch und auch literarisch vorbildhaft sind bereits die Bergbesteigungen in Bernardin de Saint-Pierres Voyage à l'île de France. Sie stehen ganz in der Traditionslinie Jean-Jacques Rousseaus und suchen vom Berggipfel aus Klarheit und Transparenz: etwas, das in der Unübersichtlichkeit der Ebenen nicht zu haben ist. Diese Bergbesteigungen mit ihrer Hervorhebung einer zunächst alpinen Bergwelt leiten über zu einer ersten Ästhetisierung nicht-europäischer Bergwelten, die uns im Reisebericht der zweiten Hälfte des 18. Jahrhunderts begegnen. Die Bergwelt und ihre Einsamkeit rücken in den Fokus des zeitgenössischen Interesses und werden in ihrer Gegensätzlichkeit zu den urbanen Räumen, aber auch zu den dicht besiedelten und bebauten Ebenen nun in ihrer Andersartigkeit akzentuiert. Und diese Akzentuierung 
bedeutet auch eine Ästhetisierung jener Bergregionen, die außerhalb dauerhafter menschlicher Siedlungen, außerhalb der Ökumene, liegen und im Grunde menschenfeindlich sind.

Vieles wäre hier beispielhaft zu erwähnen. Aber bezogen auf den Bereich spezifisch wissenschaftlicher Forschungsreisen und ihrer Ergebnisse ist einmal mehr Alexander von Humboldts Leistung zu nennen. Sie erschöpfte sich keineswegs im berühmt gewordenen Versuch einer Ersteigung des Chimborazo, ${ }^{7}$ sondern führte $\mathrm{zu}$ neuartigen Formen kartographischer Höhendarstellungen und Aufrisse, die relationstreue wie schematisierte Profile der bereisten Gebiete zeigen. Erneut ergänzen sich Auge und Ohr, werden die vom Reisenden selbst gesammelten Erfahrungen und Ergebnisse durch Resultate anderer Forscher und Reisender, durch Quellenstudien in Archiven und Bibliotheken komplettiert.

Das zweifellos berühmteste Ergebnis dieser Arbeit ist das Tableau physique des Andes et pays voisins, das Humboldt noch auf der Reise 1803 während seines Aufenthaltes in Guayaquil entworfen hatte und später in Paris zu einem auch künstlerisch beeindruckenden Werk umgestaltete. Es stellt sicherlich eines der berühmtesten Bilder der europäischen Wissenschaftsgeschichte dar - viele von Ihnen werden es bereits schon einmal gesehen haben. Naturgemälde und Idealprofil, ästhetisches Artefakt und wissenschaftliches Resultat in einem, präsentiert es eine Zusammenschau von Forschungsergebnissen, die sich auf einen weiten geographischen Raum in Abhängigkeit von den jeweiligen Höhenstufen beziehen und weit über den Gesichtskreis eines einzelnen Reisenden hinausgehen. Der Schnitt durch die Anden auf der Höhe des Chimborazo geht auf eine Zeichnung zurück, die Humboldt 1802 in seinen Amerikanischen Reisetagebüchern schematisch entwarf. Aber die ästhetische Ausführung enthält doch vieles, was sicherlich ganz neu hinzukam.

Vielleicht sollten wir zunächst einmal festhalten: Alles auf diesem Gemälde, alles in dieser Wissenschaftsskizze ist in Bewegung. In Bewegung ist die Kontinentalplatte, die hier so gezeichnet wurde, als wäre Südamerika eine Insel, die ähnlich wie der Teide auf Tenerife das Antlitz einer gesamten Landschaft

7 Vgl. hierzu Humboldt, Alexander von: Ueber einen Versuch den Gipfel des Chimborazo zu ersteigen. Mit dem vollständigen Text des Tagebuches 'Reise zum Chimborazo'. Herausgegeben und mit einem Essay versehen von Oliver Lubrich und Ottmar Ette. Frankfurt am Main: Eichborn Verlag 2016; sowie den herausragenden Film von Rainer Simon Die Besteigung des Chimborazo (Koproduktion DEFA/ZDF 1989) sowie den Band von Schäfer, Paul Kanut / Simon, Rainer: Die Besteigung des Chimborazo. Eine Filmexpedition auf Alexander von Humboldts Spuren. Köln: vgs Verlagsgesellschaft 1990. Zur ästhetischen Dimension dieser Bergbesteigung vgl. neuerdings Pimentel, Juan: El volcán sublime. Geografía, paisaje y relato en la ascensión de Humboldt al Chimborazo. In: Ette, Ottmar / Bernecker, Walther L. (Hg.): Ansichten Amerikas. Frankfurt am Main: Vervuert 2000. 


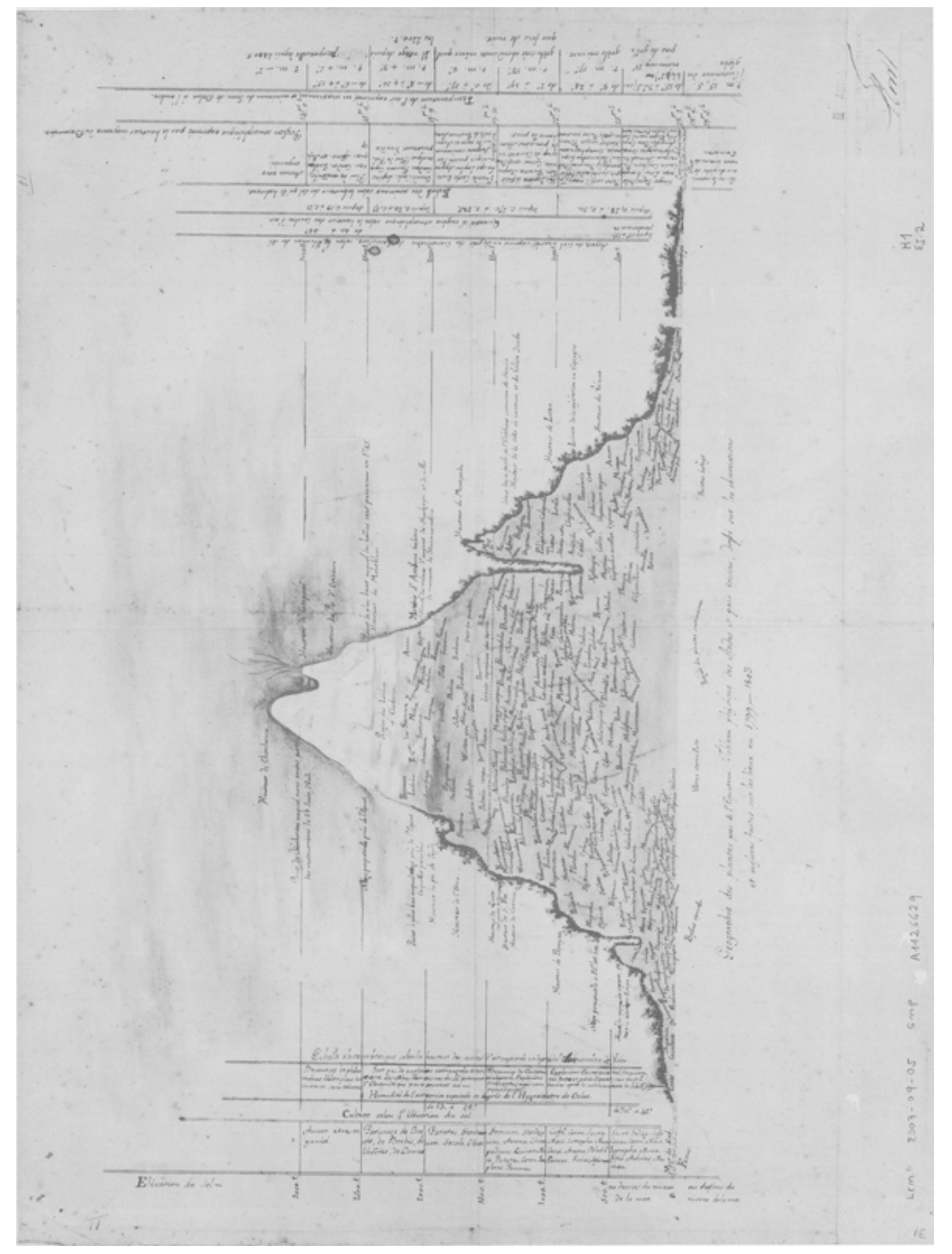

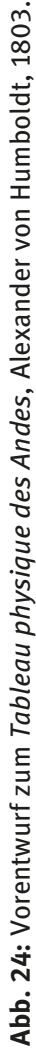




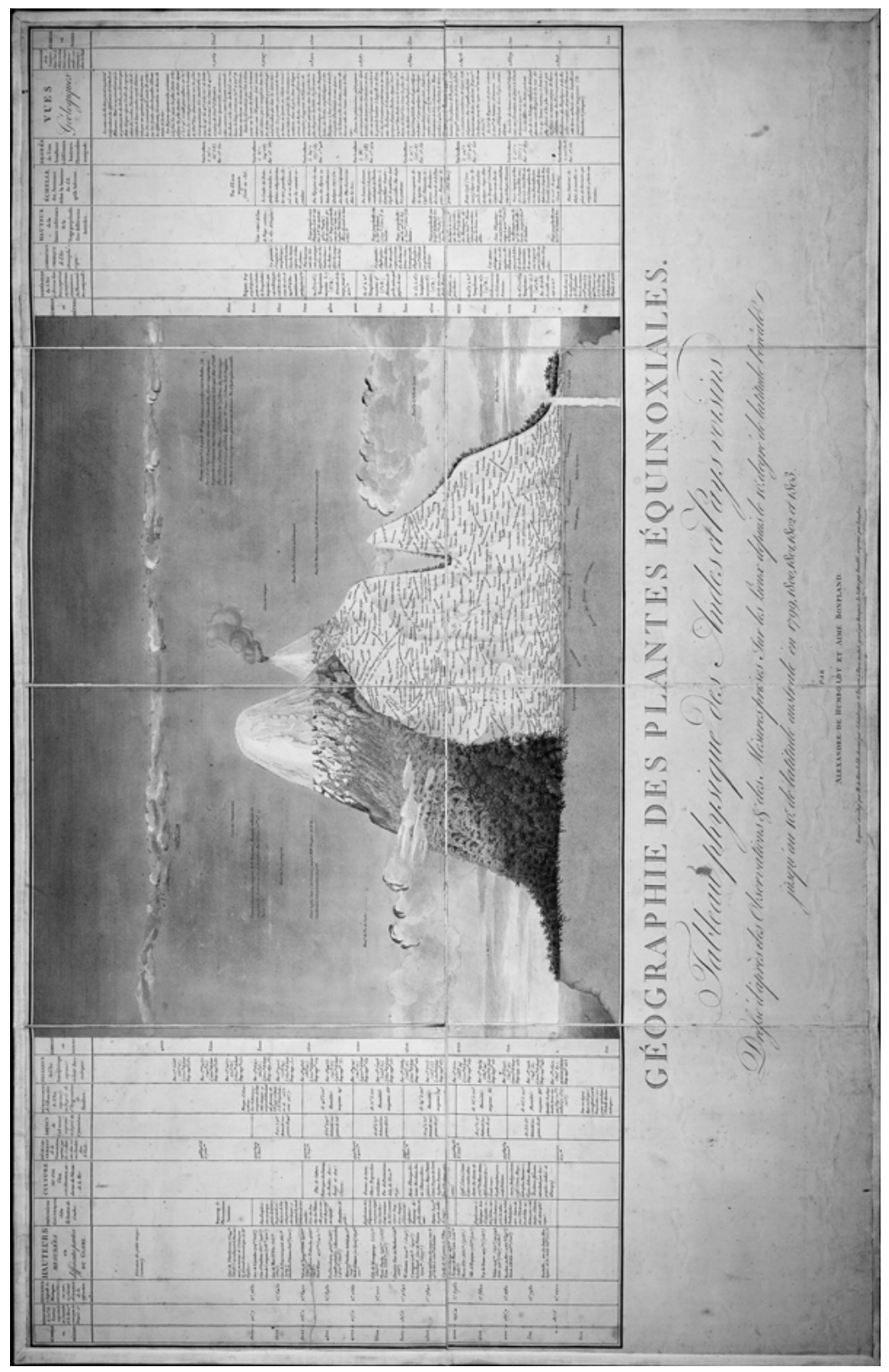

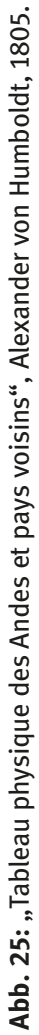


bestimmt. Lange vor Alfred Wegener hatte Humboldt verstanden, dass die Ostküste Südamerikas und die Westküste Afrikas zusammenpassten und einmal durch eine frühere 'Katastrophe' auseinandergerissen wurden. Was er noch nicht erkennen konnte, das war die Tatsache, dass sich diese Katastrophe über Jahrmillionen entfaltete und später von Wegener in einer zunächst umstrittenen Theorie als Kontinentaldrift bezeichnet wurde. Die Kontinente schwimmen gleichsam auf ihrem Untergrund und bewegen sich in einzelnen tektonischen Platten über die Erdoberfläche.

Aber auch die Geologie ist in Bewegung: die Vulkane sind aktiv, der Rauch über ihnen zeigt es deutlich an. Die verschiedenen Vegetationszonen beherbergen Gewächse, die Humboldt nicht nur in ihrem Vorkommen, sondern vor allem in ihren jeweiligen Migrationen auf der Erdoberfläche untersuchte. Seine Pflanzengeographie ist folglich eine Bewegungswissenschaft, weil er weniger eine Verteilungskarte als eine Bewegungskarte der unterschiedlichsten Gewächse anfertigt. Dazu zählen natürlich auch die künstlichen Pflanzungen tropischer Gewächse, die der Mensch angelegt hat und die in der untersten Stufe der Gewächse verzeichnet sind.

Migrationen bestimmen aber auch das Leben nicht allein der Flora, sondern auch der Fauna und insbesondere jener Tiere, die mit den Europäern nach Amerika gelangten. Und wenn Humboldt in seinen schriftlichen Kommentaren von den einzelnen Höhenstufen spricht und diese erläutert, vergisst er am Ende nicht, auch auf die von europäischen Kolonisten angelegten Plantagen und auf deren eingeführte Arbeitskräfte, die schwarzen Sklaven aus Afrika, aufmerksam zu machen. Alles in diesem Tableau physique, in diesem 'Naturgemälde', ist in Bewegung und zeichnet sich durch seine Mobilität aus.

In diesem Schnitt durch die Anden auf der Höhe von Chimborazo und Cotopaxi durchdringen sich Bild und Text wechselseitig und verweisen auf die wissenschaftsgeschichtlichen und epistemologischen Grundlagen von Humboldts wissenschaftlicher, aber auch ästhetisierender Reiseauswertung. Bildschrift und Schriftbild durchdringen sich wiederum wechselseitig. Parallel zur Zweidimensionalität der topographischen Karte zeigt auch die Profildarstellung einen Übergang von der Skizze im Reisetagebuch zur wissenschaftlichen Ansprüchen genügenden Darstellung im Idealprofil, das erneut die bereits behandelte Ausweitung von Perspektive und Blickfeld präsentiert und dabei die dritte Dimension entschieden herausarbeitet.

Zentral für diese Darstellung sind die verschiedenen Höhenstufen, welche den Aufriss charakterisieren, eine Aufeinanderfolge, deren Verschiedenartigkeit und deren Systematik Humboldt zum ersten Mal bei seinem Aufstieg auf den Teide von Tenerife überprüfen konnte. So charakterisiert sich sein Profil Südamerikas durch eine systematische Gliederung in verschiedene Höhenstufen, von der 
tierra caliente bis hin zur tierra fría der Schneeregionen. Dabei gilt es freilich hinzuzufügen, dass hier auch eine weitere Dimension in Bewegung ist, an die der preußische Reisende sicherlich nicht gedacht haben kann, weil es für derartige Vermutungen damals (noch) keinen Anlass gab. Denn wie eine Überprüfung der von Humboldt gesammelten Daten im Jahre 2012 sowie eine Publikation im Jahre 2015 ergaben, ist die Schneegrenze am Chimborazo um mehrere hundert Meter angestiegen, so dass die wissenschaftlichen Messungen zur Zeit von Humboldts Besuches der Andenriesen ihrerseits längst schon wieder historisch geworden sind. Auch hier hat der Klimawandel zu grundlegenden Veränderungen geführt und die Grenze dauerhaften Schnees erheblich ansteigen lassen - mit allen ökologischen Folgen, die daraus für die Region erwachsen.

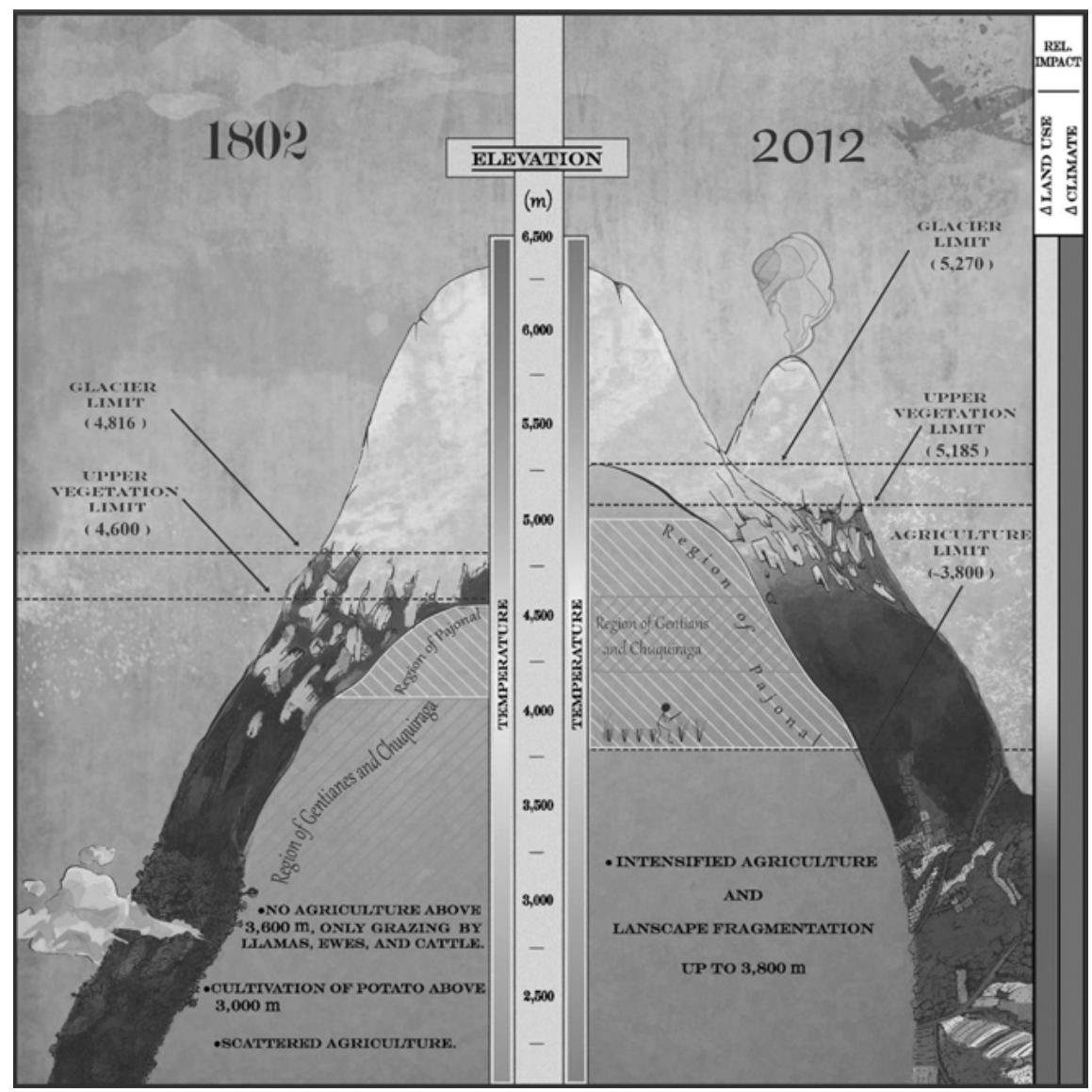

Abb. 26: ,Update‘ zu Humboldts „Tableau physique des Andes“, 1802-2012. 
In diesem Schnitt durch die Anden aber treten zugleich zwei verschiedene Orte des Schreibens einander gegenüber: Ein Ort des Schreibens während der Reise der nach Humboldts eigenen Vorgaben in späteren Kupferstichen und Gemälden dargestellt wurde - und ein zweiter Ort des Schreibens, der sich im Herkunftsland des Reisenden ansiedelt, auch dies ein Ort, der in der Ikonographie des preußischen Gelehrten mehrfach 'ausgemalt' wurde. Zwischen Humboldts 'Schreiben im Angesicht der Dinge' auf der Reise und seinen Ausführungen wie Kommentaren hierzu in Europa liegen mehrere Jahre; es kann kein Zweifel darüber herrschen, dass sich die systematische Erkenntnis Humboldts erst im Verlauf seiner weiteren Studien in Europa festigte. Die Grundprinzipien seiner Anschauung aber hatten sich schon auf der Amerikanischen Reise herausgebildet - und so findet sich das Grundaxiom seiner Wissenschaft, der Humboldt'schen Wissenschaft, auch bereits in den Amerikanischen Reisetagebüchern: „Alles ist Wechselwirkung.“8

Die vierte Dimension des Reiseberichts im Sinne von Claude Lévi-Strauss wird von der Zeit gebildet. Dabei ist die Zeit eine mobile Kategorie, die sich auf verschiedenen Ebenen des Reiseberichts einschreibt. Der Reisende bewegt sich dabei zum einen in der Zeit seines Herkunftslandes: Vergessen wir nicht, dass erst die immer zuverlässigeren Uhren es den Seefahrern des 18. Jahrhunderts erlaubten, eine immer präzisere Längenbestimmung vorzunehmen, die in einem ganz materiellen Sinne rückgebunden ist an die Ausgangszeit des Längengrades des jeweiligen Herkunftslandes. ${ }^{9}$ Der Reisende führt gleichsam seine eigene Zeit im Reisegepäck mit sich, befindet sich folglich in seiner Eigenzeitlichkeit. Raum und Zeit sind so nicht nur aufs engste miteinander verbunden, sondern zugleich an die Zeit des eigenen Herkunftsraumes rückgekoppelt. Der Reisende nicht nur des 18. Jahrhunderts ist in seiner eigenen Zeit unterwegs - und dies nicht nur mit Blick auf die Längenbestimmungen. Denn es sind gerade auch die wissenschaftlichen Diskussionshorizonte und Fragestellungen, mit denen sich der Reisende an die von ihm besuchten Regionen und Kulturen wendet, um in ihrem Umfeld nach Antworten zu suchen, welche seine Zeit ihm in seinen Herkunftsländern aufgab. die Reisenden des beginnenden

8 Humboldt, Alexander von: Reise auf dem Río Magdalena, durch die Anden und Mexico. Teil I: Texte. Aus seinen Reisetagebüchern zusammengestellt und erläutert durch Margot Faak. Mit einer einleitenden Studie von Kurt-R. Biermann. Berlin: Akademie-Verlag 1986, S. 358.

9 Zur Technik der 'horloges marines' und ihrer Bedeutung für die Seefahrt vgl. Broc, Numa: La Géographie des Philosophes: géographes et voyageurs français au XVIIIe siècle. Paris: Éditions Ophrys 1975, S. 280 ff. Eine Abweichung um nur zwei Minuten nach sechswöchiger Schiffsreise (ein Ziel, dem sich die Präzisionsinstrumente des 18. Jahrhunderts annäherten) brachte einen Fehler von einem halben Längengrad hervor (S. 282), eine gewaltige Distanz, die kartographische Verortung und späteres Auffinden von Inseln noch immer erheblich erschweren musste. 
18. Jahrhunderts stellten andere Fragen als jene, die Ende des 18. Jahrhunderts zu ihren Forschungsexpeditionen aufbrachen.

Zum anderen bewegt sich der Reisende aber auch innerhalb der eigenen Chronologie seiner Reise, die zweifellos ihre eigene Zeitlichkeit und deren Abfolgen schafft. Darüber hinaus jedoch bewegt er sich in einer Zeit, welche die von ihm besuchten Regionen charakterisiert und eine je spezifische zeitliche Umgebung prägt. Es ist nicht dasselbe, die Kolonie von Saint-Domingue vor oder nach der erfolgreichen Haitianischen Revolution zu besuchen und Haiti zu erforschen: Nichts könnte differenter sein. Die Zeit spielt eine zentrale Rolle.

Doch diese Zeit ist hochgradig heterogen und muss auf ihren unterschiedlichen Ebenen genau ins Kalkül gezogen werden. Bei ihrer bzw. seiner Reise in Raum und Zeit springt die oder der Reisende zwischen verschiedenen historischen und kulturellen Zeiten hin und her: Die Reise entwickelt nicht nur ihre Eigenzeitlichkeit, sie entwickelt auch eine Dynamik mit Blick auf die Zeit ihrer Herkunft wie gerade auch hinsichtlich jener Zeit, die das Reisegebiet auszeichnet. Dabei gilt es, diese verschiedenen Zeitlichkeiten genau im Auge zu behalten und mitzubedenken.

So versucht etwa Du Tertre ${ }^{10}$ in seinen Überlegungen zum bon sauvage, Erkenntnisse über die Entwicklung des Menschengeschlechts ausgehend von Beobachtungen in der Fremde zu gewinnen, so dass es möglich wird, Wissen über die Vorgeschichte des Eigenen durch eine Art rückwärtsgerichtete Zeitreise zu erhalten. Voraussetzung hierfür ist freilich eine Annahme von der Einheit des Menschengeschlechts und die sich daraus ableitenden Folgerungen, denen zufolge sich einzelne menschliche Gruppen jeweils schneller oder langsamer entwickelt hätten. Dies aber würde zweifellos erlauben, durch eine Reise im Raum eine Reise in der Zeit anzutreten und zu erkunden, wie denn die Vor- und Frühgeschichte der europäischen Menschheit wohl ausgesehen haben dürfte. Der bon sauvage kann in diesem Fall zu unserem Urgroßvater werden, immer vorausgesetzt, wir nehmen die These von der Einheit des Menschengeschlechtes ernst und unterscheiden nicht hierarchisch oder rassistisch zwischen verschiedenen menschlichen ‘Rassen’ oder Geschichten.

Doch ist nicht nur die Reise zeitlich rückwärts, sondern auch vorwärts in der Zeit möglich und in vielerlei Hinsicht mehr als verlockend. Wer wünschte sich nicht, einmal einen Blick in die eigene Zukunft zu werfen? So kann die eigene Gegenwart durch die Beschäftigung mit dem Anderen als künftige Vergangenheit beleuchtet werden, wenn wir diesem Anderen einen 'Vorsprung' gegenüber unserer eigenen Position zubilligen und dessen Gegenwart als unsere eigene Zukunft erkennen.

10 Vgl. Funke, Hans-Günter: ‘Barbare cruel' o ‘bon sauvage’? La funcionalización ambivalente de la imagen del indio en la 'Histoire générale des Antilles' (1667-1671) del Padre du Tertre. In: Dispositio (Ann Arbor) XVII, 42-43 (1992), S. 73-105. 
So kann die Reise in ein Land, das unsere eigene künftige Gesellschaft vorwegnimmt, zu einer Reise in unsere eigene mögliche Zukunft werden. Wir können gleichsam hautnah erleben, was unserer Gesellschaft künftig bevorsteht und welchen Übeln wir möglicherweise begegnen müssen. Beispielsweise erkundete Alexis de Tocqueville in seinem grundlegenden Werk De l'Amérique, das auf einer Nordamerikareise des Jahres 1831 beruhte, jene Möglichkeiten, Risiken und Chancen, welche die demokratische Verfassung Nordamerikas für die europäischen Staaten und insbesondere Frankreich bereithielt, welche also jene künftigen Aspekte seien, die man erhoffen dürfe oder befürchten müsse. ${ }^{11}$ Einen wichtigen Ausgangspunkt stellt dabei eine schlichte Frage dar: 'Wohin führt uns unsere Reise?'

\begin{abstract}
Glaubt man etwa, dass die Demokratie, nachdem sie den Feudalismus zerstört und die Könige besiegt hat, vor den Bürgern und den Reichen zurückweichen wird? Wird sie nun, da sie selbst so stark und ihre Gegner so schwach geworden sind, einfach stehenbleiben? Wohin gehen wir also? Niemand wüsste dies zu sagen; denn uns fehlen bereits die Vergleichspunkte: Die Lebensumstände (conditions) sind heutzutage unter den Christen gleicher, als dies je zu einer anderen Zeit oder in einem andren Land der Welt der Fall war; so verhindert die Größe dessen, was schon getan ist, die Voraussage dessen, was noch getan werden kann. [...] Es tut nicht not, dass Gott selbst spricht, damit wir sichere Zeichen seines Willens erhalten; es genügt zu untersuchen, welches der gewöhnliche Gang der Natur und welches die beständige Tendenz der Ereignisse ist. ${ }^{12}$
\end{abstract}

Man hört gleichsam die behutsame Frage von Denis Diderot in Jacques le fataliste et son maître heraus: 'Est-ce que l'on sait oú l'on va?' Die epochenspezifische Erfahrung einer historischen Entwicklung, die sich zunehmend den bekannten Vorbildern entzieht und gerade im nachrevolutionären Frankreich der Historia als Magistra Vitae jegliche Legitimation abspricht, ${ }^{13}$ führt hier - die Formel Où allons-nous donc? scheint es bereits anzudeuten - zu einer Ausweichbewegung im Raum: Eine Untersuchung der Demokratie in den Vereinigten Staaten soll Aufschluss geben über deren Entwicklung in Europa. Fürwahr eine Wette auf die Zukunft - und eine erfolgreiche allemal, denn Tocqueville hat mit seiner Betrachtung der Vereinigten Staaten von Amerika als Behältnis der Zukunft Europas Schule gemacht. Erst vor wenigen Jahrzehnten haben wir das von Tocqueville skizzierte Paradigma verlassen, wobei wir durchaus noch immer in manchen

11 Vgl. auch Neumeister, Sebastian: Alexis de Tocqueville. In: Lange, Wolf-Dieter (Hg.): Französische Literatur des 19. Jahrhunderts. Bd. II. Heidelberg: Quelle \& Meyer 1980, S. 85.

12 Tocqueville, Alexis de: De la démocratie en Amérique. Première édition historico-critique revue et augmentée par Eduardo Nolla. Bd. 1. Paris : Librairie philosophique J. Vrin 1990, S. 8. 13 Vgl. auch Koselleck, Reinhart: Historia Magistra Vitae. Über die Auflösung des Topos im Horizont neuzeitlich bewegter Geschichte. In (ders.): Vergangene Zukunft. Zur Semantik geschichtlicher Zeiten. Frankfurt am Main: Suhrkamp ${ }^{2} 1984$, S. 38-66. 


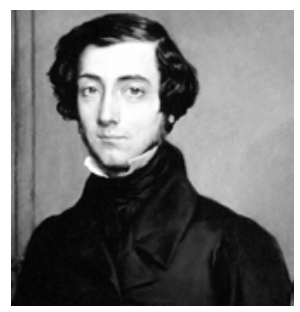

Abb. 27: Porträt von Alexis de Tocqueville (Verneuil-sur-Seine, 1805 - Cannes, 1859).

Bereichen nach den USA schielen, um besser zu verstehen, welche Gefahren uns und unseren Demokratien in Europa drohen könnten. Die Überwachungsdemokratien der Massenkonsumgesellschaft in den USA sind uns nicht erst seit Edward Snowdon in ihren Reichweiten und Konsequenzen höchst vertraut. Und auch der Populismus eines Donald Trump nimmt einiges an möglichen Entwicklungen unserer Demokratien vorweg, die uns sehr wohl massiv bedrohen könnten. Insofern gilt noch immer: Est-ce que l'on sait où l'on va? Aber die USA sind glücklicherweise nicht mehr der entfernte Spiegel, in dem wir unsere Zukunft betrachten können. Und der von Europa gewählte Weg ist ein anderer.

Bei Alexis de Tocqueville jedenfalls wird die Reise gen Westen zu einer politischen Zeitmaschine, die der französische Reisende und Politiker wohl als erster in einer langen, bis heute andauernden Reihe von Reisenden in Gang setzte. Die Reise auf einen anderen Kontinent wird zu einer Reise in ein Land der Zukunft, die Vereinigten Staaten von Amerika. Andere Projektionen stehen dahinter zurück; vergessen wir etwa nicht Stefan Zweig, der in seinem Buch aus dem brasilianischen Exil Brasilien als das Land der Zukunft beschwor. Die Versuchung ist immer allen europäischen Intellektuellen nahe, die Reise im Raum zu einer Reise in der Zeit zu machen. Derzeit aber können wir nur hoffen, dass die Entwicklungen in Brasilien, das sich deutlich in Richtung einer Diktatur bewegt, nicht für die Zukunft unseres Kontinents stehen werden.

Doch kehren wir wieder enger zurück zur vierten Dimension unseres Reiseberichts: der Zeit. Sind deutsche oder italienische Reiseberichte der Nachkriegszeit aus und über die Vereinigten Staaten in dieser Traditionslinie nicht auch häufig Erkundungsreisen gewesen, welche sich weniger um ein Verständnis der aktuellen Bedingungen des Fremden als um eine Reflexion der künftigen Möglichkeiten des Eigenen bemühten? So kann die Reise im Raum - ganz so, wie der kubanische Romancier Alejo Carpentier dies in seinem Orinoco-Roman Los pasos perdidos ausdrückte $^{14}-\mathrm{zu}$ einer Reise in verschiedenen Zeiten und zu verschiedenen Epochen

14 Vgl. Carpentier, Alejo: Los pasos perdidos. Madrid: Ediciones Akal 2009. 
werden, eine Reiseform, die ähnlich wie beim Umspringen der Utopie in die Uchronie dem Reisenden des ausgehenden 18. Jahrhunderts in ihren Möglichkeiten bezüglich der hier nur angedeuteten Offenheit der Zukunft wesentlich bewusster geworden war. Die USA boten eine willige Projektionsfläche für jene Welt, die sich nunmehr als die Alte begriff. Der Blick in die Neue Welt schien eine neue Welt heraufzuführen. Auch hier steht - nicht anders als in unserem Jahrhundert - Reiseliteratur für Literatur schlechthin ein. Wir sollten uns aber wünschen, die Zukunft Europas nicht mehr in den USA zu sehen, sondern einen Weg zu beschreiten, der deutlich einer des Viellogischen ist - einer Polylogik, innerhalb derer sich unterschiedliche Entwicklungen in Europa ergeben und reifen können.

Die europäischen Reisenden des 18. und wohl auch noch jene des 19. Jahrhunderts glauben allerdings an eine gemeinsame Zeit der Menschheit, eine Zeitachse also, auf die sich die von ihnen konstatierten verschiedenen Zeitebenen linear beziehen lassen. Wir bewohnen sozusagen dieselbe Zeit, nur befinden wir uns an unterschiedlichen Punkten desselben Zeit-Raumes.

Bei einer derartigen Vorstellung wird die Zeitreise notwendigerweise zur Bewegung des Reisenden zwischen verschiedenen Stufen kultureller, historischer, ökonomischer und sozialer Entwicklung, unabhängig davon, ob diese Entwicklung positiv oder negativ eingefärbt, ob die Entwicklung folglich als Höherentwicklung oder als Degradation gelesen und eingeordnet wird. Die Entdeckung voneinander unabhängiger, partikulärer Zeiten gewinnt (soweit ich sehe) erst in der Reiseliteratur des 20. Jahrhunderts an Raum - zusammen mit Vorstellungen, welche die unterschiedlichen Logiken verschiedenartiger Räume und damit ein polylogisches System denken können. Aber so weit sind wir im 19. Jahrhundert noch nicht.

Auch Flora Tristans Reise nach Peru, ihre berühmten Pérégrinations d'une paria, lässt die Erfahrung der Zeitreise nicht vermissen, glaubt sich die Verfasserin des bis heute faszinierenden Reiseberichts doch etwa angesichts der von ihr dargestellten 'Mysterienspiele' in Arequipa ins europäische Mittelalter versetzt. Wir sind bei ihr also unterwegs auf derselben Zeitachse, nun aber in rückwärtsgewandter Vektorizität, so dass wir eine Reise in der Zeit nach hinten antreten. Hören wir die mit frühsozialistischen Ideen arbeitende Kämpferin für die Emanzipation der Frauen nicht nur in Frankreich, sondern auf der ganzen Welt:

Für mich, ein Kind des 19. Jahrhunderts und aus Paris kommend, war die Aufführung eines Mysterienspiels unter dem Portal einer Kirche und vor einer unermesslich großen Volksmenge etwas Neues; aber das lehrreiche Schauspiel waren die Brutalität, die grobe Kleidung, die Lumpen eben dieses Volkes, dessen extreme Unwissenheit, dessen dummer Aberglaube meine Einbildungskraft ins Mittelalter zurückführten. Alle diese weißen, schwarzen oder kupfernen Figuren drückten eine ungezähmte Wildheit, einen übersteigerten Fanatismus aus. Das Mysterienspiel ähnelte ziemlich, durch seinen Hintergrund (ich werde nicht von den Schönheiten des Dialogs sagen, denn die Worte haben nur unvollkommen mein Ohr 
erreicht), denjenigen, die im fünfzehnten Jahrhundert vorgeführt wurden, mit großen Pomp, im Saal des Justizpalastes, zur Erbauung des guten Volkes von Paris, eine Vorstellung, an der uns Victor Hugo in seinem Notre-Dame teilnehmen lässt. ${ }^{15}$

Flora Tristan (vgl. Abb. 81: Porträt der Flora Tristan) zieht aber nicht nur den Vergleich mit einem Europa des Mittelalters, einem Europa eines wohlgemerkt dunklen Mittelalters, in welchem Aberglaube und Fanatismus im Zeichen der Katholischen Kirche herrschten, sondern zieht auch eine Verbindung mit einer literarischen Veranschaulichung des französischen Mittelalters in Gestalt jenes Romans, in welchem uns Victor Hugo seine Sichtweise der dunklen Zeit vor der Frühen Neuzeit und damit den temps modernes in einem breiten literarischen $\mathrm{Pa}$ noramabild entfaltete. Notiert die französische Reisende, die aus Frankreich buchstäblich geflohen war und mit großen Erwartungen in die Neue Welt kam, auch sehr genau, wie schnell die französische Mode die Toilette der peruanischen Frauen diktiert, kommt sie doch nicht umhin, aus dem, was sie als Aberglauben bezeichnet, den Schluss zu ziehen, dass das peruanische Volk noch in seiner Kindheit ${ }^{16}$ verharre und auch noch lange der Kirchenmacht ausgeliefert bleiben werde. Der literarische Bezugspunkt für Floras Darstellung eines Mysterienspiels blieb freilich nicht ungenannt: Die Erzählerin selbst verweist auf Victor Hugos Notre-Dame de Paris, ${ }^{17}$ das nur wenige Jahre zuvor erschienen war. Wir haben es also mit einem durch und durch literarischen Mittelalterbild $\mathrm{zu}$ tun, das wohl kaum unserem zeitgenössisch differenzierten Bild des Mittelalters entsprechen dürfte. Aber für Flora Tristan war dies der 'ferne Spiegel', ${ }^{18}$ in dem sich die damalige Jetztzeit aus der zeitlichen (und räumlichen) Ferne betrachtete.

Fassen wir also beherzt zusammen: Alexis de Tocquevilles Reise von 1831 in die USA führt den Franzosen in die Zukunft, Flora Tristans Reise von 1833 nach Peru die Französin dagegen in die Vergangenheit. Wir haben es hier mit einer umgekehrten Vektorizität zu tun, insoweit in beide Reisen ein entgegengesetzter Zeitpfeil eingetragen ist. Bei beiden jedoch wird das Andere, die Zeit des Anderen, auf die eigene Zeit und deren Chronologie bezogen - ein interessantes chassé-croisé, das noch dadurch an Reiz gewinnt, dass beide höchst unterschiedlichen, an der Vergangenheit beziehungsweise der Zukunft orientierten Wertvorstellungen anhingen - hier, etwas verknappt ausgedrückt, der konservativ-monarchistische

15 Tristan, Flora: Les Pérégrinations d'une paria 1833-1834. Paris: La Découverte 1983, S. 143 f.

16 Ebda., S. 130: „So sind die Völker in der Kindheit“.

17 Ebda., S. 144.

18 Vgl. Tuchmann, Barbara: Der ferne Spiegel. Das dramatische 14. Jahrhundert. Übersetzung Ulrich Leschak und Malte Friedrich. Düsseldorf: Claassen 1980. 
Tocqueville, dort die frühsozialistische Kämpferin für eine künftig gleichere Gesellschaft im Zeichen einer grundlegenden Emanzipation.

Wir befinden uns folglich im Reich der Zeit. Die vierte Dimension beinhaltet die Koexistenz, das Ineinanderspielen verschiedener Zeitachsen und Zeitvorstellungen unter Einbeziehung der damit verbundenen (geographischen, kulturellen, politischen oder sozialen) Räume. Die Konfrontation verschiedener Zeitebenen trägt wesentlich zum Reiz und zur Attraktivität des Reiseberichtes und jeder Literatur bei, die sich in Bewegung befindet. Dazu zählt auch und gerade die Reiseliteratur.

Die von dem französischen Anthropologen Claude Lévi-Strauss noch angesprochene fünfte Dimension der Reise betrifft die gesellschaftliche, die soziale Dimension. Die oder der Reisende bewegt sich durch die verschiedenen sozialen Gruppen und Schichten des von ihr oder ihm bereisten Landes oft mit einer Leichtigkeit, die dem Einheimischen, insbesondere in den stärker hierarchisierten Gesellschaften des 18. und 19. Jahrhunderts, weitgehend unzugänglich bleibt. Dies bedeutet, dass im literarischen Reisebericht oftmals ein Gesamtbild einer Gesellschaft entsteht, die in ihren unterschiedlichen Schichten fundamental gequert und veranschaulicht wird. Die Reisenden bewegen sich nicht allein in Raum und Zeit, sondern bewegen sich in Gesellschaften, die möglichst breit darzustellen sie angetreten sind. Sie wollen alles präsentieren: die gesellschaftlichen Spitzen wie auch all jene, die den 'Bodensatz' einer Gesellschaft darstellen. Auch dafür gibt es literarische Modelle.

Dies kommt auch in den unterschiedlichen Anlagen der Reiseberichte zum Ausdruck. Die Französin Flora Tristan, die im Gegensatz zur vorherrschend naturkundlichen Ausrichtung des Reiseberichts über Lateinamerika eine wesentlich politischere Orientierung verfolgt und durch ihre familiären Beziehungen Zugang auch zu höchsten Schichten der jungen peruanischen Republik erhielt, kann in dieser Hinsicht aus dem Vollen schöpfen und macht nicht von ungefähr ein breites soziales Panorama zur Vorbedingung jedweder Darstellung, die legitimen Abbildanspruch erheben will. ${ }^{19}$ Es ist der Anspruch, eine ganze Gesellschaft, eine Gesellschaft in ihrer Totalität zu erfassen und zu porträtieren.

Der neuspanische Dominikanermönch Fray Servando Teresa de Mier lernt Angehörige unterschiedlichster Schichten der spanischen Gesellschaft ebenso intensiv kennen wie sein Zeitgenosse Alexander von Humboldt bei seiner

19 Vgl. Tristan, Pérégrinations, S. 85: „um eine Stadt darzustellen, auch wenn sie nur ein wenig Wichtigkeit besitzt, muß man sich längere Zeit in ihr aufhalten, mit allen Klassen ihrer Bewohner sprechen.“ 
Reise durch Neu-Spanien, ${ }^{20}$ dem späteren Mexiko. Beide bemühen sich mit Hilfe unterschiedlicher Mittel, eine Gesellschaft in ihrer Totalität zu erfassen und darzustellen. Doch wie gesagt: Der Reisebericht rückt damit in die Nähe eines wichtigen literarischen Genres, das gerade auch den Berichten des rebellischen und aufmüpfigen Mönches Fray Servando nicht fern ist: Ich meine den Schelmenroman, die Novela picaresca, die im Übrigen zeitgleich just in Neu-Spanien, dem heutigen Mexico, mit Fernández de Lizardis El Periquillo Sarniento zu Beginn des 19. Jahrhunderts den Weg der spanischen Kolonialliteraturen in die Romanwelt der modernen lateinamerikanischen Literaturen buchstäblich eröffnete. Die moderne Erzählliteratur eines unabhängigen, von den kolonialen Fesseln befreiten Mexiko beginnt mit einer Novela picaresca, mit einem Schelmenroman, welcher nicht nur die Gesellschaft und ihre sozialen Bedingungen, sondern auch deren räumliche Diversität und Heterogenität auf einzigartige Weise darstellte. Hier wird ein literarisches Genre, das an der Wiege des europäischen Romans stand und gleichsam die modern times, die Neuzeit eröffnete, in die Lage versetzt, den Abbildanspruch jener Gattung einzulösen, die das 19. Jahrhundert beherrschen sollte: der Roman, der mit Hegel als ‘bürgerliche Epopöe' stets in der Pflicht steht, eine gesellschaftliche Totalität zur Abbildung zu bringen und nichts in dieser Totalität unberührt zu lassen. So ist es faszinierend, dass der Schelmenroman nicht nur an der Wiege des ersten modernen Romans zu Beginn der Neuzeit, Miguel de Cervantes' Don Quijote, sondern auch an jener des bürgerlichen Romans stand, wie er sich im Verlauf des 19. Jahrhunderts herausbilden sollte. Sein Auftrag: eine gesamte Gesellschaft in literarischer mimesis abzubilden.

Die unablässigen Bewegungen zwischen den Höhen und Tiefen einer Gesellschaft, das ständige Auf und Ab in der fremden Sozietät bieten gerade dem Reisenden des 19. Jahrhunderts die Möglichkeit, in Konkurrenz zum historischen Roman eines Walter Scott oder zum realistischen Romanmodell eines Balzac zu treten und aus der eigenen (hermeneutischen) Bewegung ein gesamtgesellschaftliches Panorama einzufangen. Darstellbar werden nicht nur die sogenannten 'höheren Stände', die gesellschaftliche Elite eines Landes mit ihren verfeinerten Sitten und ihren selbstverständlichen Bereicherungen, sondern auch all jene, die in finsteren Kerkern eingesperrt sind, die als Sklaven oder billige Lohnarbeiter ein entbehrungsreiches Leben führen oder diejenigen, welche aus der Gesellschaft ‘ausgestiegen' sind und als Vagabunden über Land ziehen

20 Vgl. Ette, Ottmar: Transatlantic Perceptions: A Contrastive Reading of the Travels of Alexander von Humboldt and Fray Servando Teresa de Mier. In: Dispositio (Ann Arbor) XVII, 42-43 (1992), S. 165-197. 
und eigenartige Parallelen zu den Reisenden selbst erlauben. Nicht umsonst bezeichnete sich Flora Tristan als eine aus der Gesellschaft Ausgestoßene, als eine Paria, die in der Gesellschaft ihrer Zeit keinen Platz mehr habe.

Doch der Reisebericht in seiner Nähe zur Novela picaresca kann noch mehr. Denn zugleich kann der bürgerliche Roman insoweit überboten werden, als der Abbildanspruch durch den Verweis auf den Augenzeugenstatus des Berichtenden und durch die Faktizität nachprüfbarer Reisewege untermauert wird. Die oder der Reisende kann mit einer hohen Glaubwürdigkeit rechnen und als gesicherter Augenzeuge auftreten. Leicht referentialisierbare Örtlichkeiten und beigegebene Karten verschaffen dem Leser einen sicheren, präzise Faktentreue vorspiegelnden Rahmen für die Lektüre des Textes, der uns vorgaukelt, die Wirklichkeit so abzuspiegeln, wie sie wirklich ist. Reiseliteratur ist nicht zuletzt jene Literatur, die das Lesepublikum zumindest insoweit in Bewegung setzt, als sie dieses auffordert, auf einschlägigen topographischen Karten die dargestellten Reisewege 'nachzufahren' und nachzuvollziehen. Sie scheint uns damit die aufwendige Ökonomie der Fiktion, des Erfundenen, zu ersparen. Aber dies, so haben wir schon zuvor gesehen, ist in keiner Weise der Fall.

So kann folglich kein Zweifel daran bestehen, dass diese fünfte durch eine sechste Dimension ${ }^{21}$ ergänzt werden muss, jene der Imagination und Fiktion, welche den Reisebericht gerade im Rückgriff auf fiktionale literarische Muster für den jeweiligen zeitgenössischen Leser und vielleicht mehr noch die am Reisen oft gehinderte zeitgenössische Leserin attraktiv und lesbar macht. Die Fiktion ist gleichsam das reiseliterarische Schmiermittel, auf dem die Wege des Wissens im Reisebericht wesentlich besser gleiten und gerade auch in ästhetischer Hinsicht alle Formen und Formate des Reiseberichts weitaus überzeugender (und bisweilen eleganter) daherkommen.

Fiktion ist aber auch die vielleicht entscheidende Verstehenshilfe reiseliterarischer Bewegungen, erlaubt sie es doch, einen Bezugsrahmen für das im Reisebericht Vorzustellende herzustellen. Auch Alexander von Humboldt sah am Ende seines historischen Rückblicks auf die 'Anregungsmittel zum Naturstudium' in seinem Kosmos im Jahre 1847 keinen Gegensatz zwischen der wissenschaftlichen und einer spezifisch poetischen Dimension und Funktion des Reiseberichts. Er schrieb der Funktion eine belebende Wirkung zu, mit anderen Worten: Erst die Fiktion haucht dem Berichteten Leben ein. Aber hören wir Humboldt selbst:

21 Vgl. auch Pagni, Andrea / Ette, Ottmar: Introduction. In (dies., Hg.): Crossing the Atlantic: Travel Literature and the Perception of the Other. Doppelnummer der Zeitschrift Dispositio (Ann Arbor) XVII, 42-43 (1992), S. iv. 
Naturbeschreibungen, wiederhole ich hier, können scharf umgrenzt und wissenschaftlich genau sein, ohne daß ihnen darum der belebende Hauch der Einbildungskraft entzogen bleibt. Das Dichterische muß aus dem geahndeten Zusammenhange des Sinnlichen mit dem Intellectuellen, aus dem Gefühl der Allverbreitung, der gegenseitigen Begrenzung und der Einheit des Naturlebens hervorgehen. ${ }^{22}$

Das zentrale Lexem in diesem Zusammenhang ist das Wörtchen 'Leben'. Die Lebendigkeit des Berichteten wird erst durch die Fiktion im Berichteten selbst hergestellt. Dabei handelt es sich um ein Zusammenspiel. Dies aber meint, dass der Fiktion eine zugleich funktionale und dienende Rolle zugeschrieben wird. Dem Zusammenspiel von wissenschaftlicher Genauigkeit und dichterischer Einbildungskraft im Reisebericht soll vertieft in den Überlegungen zur Friktionalität des Genres nachgegangen werden.

Doch schon hier kann festgehalten werden, dass es nicht allein die intellektuelle Seite ist, die im Vordergrund des Reiseberichts steht. Vielmehr ist in der Sinnlichkeit des Berichteten eine Qualität vorhanden, die der Anschaulichkeit für das Lesepublikum vermöge der Einbildungskraft der Schriftstellerin oder des Schriftstellers auf die Sprünge hilft. Humboldts Wissenschaft ist eine sinnliche Wissenschaft, die nicht in der Vermessung der Welt ihren Seinsgrund besitzt, sondern Natur und Kultur in ihrer Darstellung sinnlich erfahrbar machen möchte. Dazu aber bedarf sie der Einbildungskraft, dazu bedarf sie der Fiktion, die es dem empirisch Festgestellten erlaubt, in seiner Sinnlichkeit wahrgenommen werden zu können. Die Humboldt'sche Wissenschaft ist in diesem Sinne eine sinnliche und insoweit - ganz im Sinne Friedrich Nietzsches - eine alle Sinne bedienende fröhliche Wissenschaft.

Doch kommen wir nun zu einer Dimension, die von alledem grundsätzlich nicht unabhängig ist, sondern eine tiefe Verbindung mit dieser sechsten Dimension eingeht. Die überaus komplexe siebte Dimension des Reiseberichts ließe sich als jene des literarischen Raumes bezeichnen, ein Terminus, den wir sogleich definieren wollen. Sie betrifft die Art und Weise, wie ein bestimmter Reisebericht sich $\mathrm{zu}$ anderen Texten anderer Autoren (also intertextuell) oder auch zu eigenen Texten derselben Autorin oder desselben Autors (mithin intratextuell) in Beziehung setzt. Diese Beziehung kann paratextuell hergestellt und erzeugt werden, aber auch im eigentlichen reiseliterarischen Text selbst generiert sein. Gerade die paratextuellen Verweise - also jene, die gleichsam an den Schwellen eines Textes, in seinem Titel, seinen Vorworten, seinen Fußnoten

22 Humboldt, Alexander von: Kosmos. Entwurf einer physischen Weltbeschreibung. Hg. von Ottmar Ette und Oliver Lubrich. Frankfurt a.M.: Eichborn 2004, S. 223-224. 
und vielem anderen mehr - sind es, welche die intertextuelle Relationalität eines bestimmten reiseliterarischen Textes explizit in Szene setzen.

Dabei kann zwischen einem expliziten und einem impliziten literarischen Raum unterschieden werden, insoweit andere Texte durch direkte Verweise oder durch indirekte Anspielungen, die nicht für alle Leser sofort durchschaubar sind, in den eigenen Text 'eingeblendet' werden. Dass Humboldts Ansichten der Natur etwa dem Schreiben von Georg Forster, des Begleiters von James Cook auf dessen zweiter Weltumsegelung, sehr viel verdanken, drückt sich nicht nur in der Struktur und der Schreibweise, sondern auch bereits im Titel des Werkes aus, pflegt dieser doch bewusst eine Beziehung zu Forsters Ansichten vom Niederrhein. So kann ein Text einen anderen aufrufen und zugleich verbergen und kaschieren: un texte peut en cacher un autre.

Gerade expliziten Verweisen kommt oftmals eine diskursstützende, legitimatorische Funktion zu. Ein bestimmter Text begründet seine Existenz mit Verweis auf einen anderen, vom Lesepublikum bereits begeistert aufgenommenen und kanonisierten Text, der gleichsam als Bezugssystem und damit als Rechtfertigung dient. Aufschlussreich und bedeutsam ist zum Beispiel die Frage, ob sich ein(e) europäische(r) Reisende(r) nicht nur auf Berichte eigener Landsleute, sondern auch auf Texte, die von Bewohnern der bereisten Gebiete stammen, bezieht und damit seine außereuropäischen Quellen aufwertet. Für lange Zeiten waren positive Bezugnahmen auf die 'Gegenstände', also die Objekte des Reiseberichts in der europäischen Tradition des 18. Jahrhunderts nahezu tabu, konnte der europäische Reisende doch nichts - etwa von den indigenen Völkern über diese selbst - in Erfahrung bringen, was für die Redaktion seines nach europäischen Kriterien abgefassten Reisebericht von Belang gewesen wäre. Allein die Stimme des europäischen Reisenden war mit jener Autorität und Glaubwürdigkeit ausgestattet, die dem europäischen Lesepublikum vertraut waren.

Dieser Aspekt der siebten Dimension beinhaltet stets auch die Frage, inwieweit die Objekte des Reiseberichts selbst auch als Subjekte zu Wort kommen (dürfen). Der europäische Reisende hört zwar die verschiedenen Stimmen der 'Eingeborenen', die zu ihm sprechen; er kennt zwar die Werke einheimischer Autoren, die über bestimmte Sitten und Gebräuche Aufschluss geben; aber er selbst wählt aus, was an diesen Stimmen in seinem Bericht hörbar werden kann und verleibt diese Elemente dann seiner eigenen Stimme ein. Dem Objekt ist es somit nicht gelungen, $\mathrm{zu}$ einem eigenen Subjekt $\mathrm{zu}$ werden: Es verharrt in seiner $\mathrm{Ob}$ jekthaftigkeit und wird nur als Gegenstand dargestellt, stellt sich selbst im Bericht des europäischen Reisenden aber nirgendwo dar. Es wird re-präsentiert, präsentiert sich aber nirgendwo selbst. Seine Stimme wird nicht hörbar, sie wird bestenfalls der Stimme des oder der Reisenden einverleibt und geht als Stimme eines eigenständigen Subjekts, das für sich selbst sprechen kann, verloren. Ein 
grundlegender Wandel lässt sich diesbezüglich ungeachtet aller Vorläufer definitiv erst im 20. Jahrhundert auf breiterer Front erkennen, werden nun doch auch die Bereisten selbst in die Sinnbildungsprozesse etwa europäischer Reisender miteinbezogen. Das fremde Objekt, der fremde Gegenstand erhält erst spät seine Stimme; und ob sie gehört wird, steht noch auf einem anderen Blatt. Koloniale Präsentations- und Wahrnehmungsmuster dominieren über lange Jahrhunderte und sie sind bis heute keineswegs verschwunden.

Eine achte Dimension des Reiseberichts betrifft die genrespezifischen Bezüge, wobei hier nicht mehr die Frage untersucht wird, auf welche norm- oder vorbildgebenden Einzeltexte sich der analysierte Reisebericht bezieht, sondern welche literarischen Gattungen, Subgattungen und Traditionen, welche wissenschaftlichen und insbesondere naturwissenschaftlichen Bezugssysteme Berücksichtigung finden und inwieweit Genealogien von Reiseberichten in den jeweiligen Text eingearbeitet wurden. Anders als in Hinblick auf den literarischen Raum geht es folglich nicht mehr um Einzeltexte und Autoren, sondern um Gruppen von Texten, die sich zu Gattungen, Subgattungen oder anderen genremarkierten Textsorten formieren.

Denn die auf den empirischen Raum bezogene Gattung des Reiseberichts, aber auch die Literatur insgesamt, nimmt stets bestimmte Positionen innerhalb spezifisch literarischer und gattungsgeschichtlicher Räume ein, verortet sich also selbst innerhalb ihres je eigenen mappings. $\mathrm{Zu}$ den für das 18. Jahrhundert entscheidenden Subgattungen zählt zweifellos die 'Reise um die Welt', die von großer Strahlkraft wurde. Sie setzt den Blick des europäischen Reisenden auf seine außereuropäischen Objekte absolut und füllt gleichsam riesige Bereiche eines Hinterlandes, des niemals erkundet wird. Die europäischen Weltumsegelungen des 18. Jahrhunderts berührten in der Regel stets nur Hafenstädte und Küstensäume, formulierten aber Einschätzungen von ganzen Ländern, die niemals vom Reisenden selbstständig in Augenschein genommen wurden. Die Reise um die Welt, die mit Georg Forsters gleichnamigem Werk in englischer und deutscher Sprache einen kanonischen Status erreichte, bildete das vorbildgebende Modell auch für Beschreibungen, welche sich einzelnen Zielregionen zuwandten, ohne jeweils eine eigentliche Weltumsegelung darzustellen.

Steht in dieser Tradition des Reiseberichtes der eigentliche Weg, das Itinerarium, im Vordergrund, so gibt es vor allem seit der zweiten Hälfte des 20. Jahrhunderts Reiseberichte, in welchen der eigentliche Weg als weitestgehend oder sogar solcher vollständig verschwindet. Wir hatten dies bereits in Michel Butors Mobile beobachten können, wo wir es mit diskontinuierlichen Sprüngen zwischen einzelnen States der Vereinigten Staaten zu tun hatten. Wir reisen hierbei nicht durch ein bestimmtes Territorium, sondern 
durch ein Alphabet, werden die Staaten doch in ihrer alphabetischen Anordnung durchreist und abgehandelt.

Wir erkennen dieses Schema einer Aufgabe des Itinerarischen aber auch in den Reiseberichten eines französischen Landsmannes von Michel Butor, nämlich in den Büchern von Roland Barthes, des Zeichentheoretikers und Semiologen, der als Schriftsteller gewiss ein enfant terrible seiner Zunft war und ein Vertreter dessen, was seine Gegner als die terreur de la théorie brandmarkten. Er versammelte beispielsweise in seinen Incidents verschiedene Marokko-Reisen und in seinem L'Empire des signes seine unterschiedlichen Japan-Aufenthalte, ohne auch nur den Versuch zu unternehmen, die verschiedenen Reisen voneinander zu unterscheiden oder gar zu datieren. Bis auf wenige Restbestände sind Reisewege aus dieser Art experimentellen Reiseberichts getilgt: Der Reisende reist nicht, er ist einfach vor Ort und entfaltet einen anthropologischen Blick, in dessen Blickwechsel freilich stets andere, 'einheimische' Blicke eingehen und sinnlich erfahrbar werden. Dass die Fiktion in diesem Zusammenhang eine wichtige Rolle spielt, versteht sich innerhalb unserer Argumentation beinahe von selbst.

Dieser experimentelle Modus reiseliterarischen Reise-Schreibens hat durchaus Schule gemacht und versuchte, den kolonialen europäischen Blick auf 'seine' Gegenstände zu unterlaufen und einer grundlegenden Kritik zu unterziehen. So ging es Roland Barthes explizit um eine „dépossession de l'Occident“, ${ }^{23}$ um eine Selbst-Enteignung des Abendlandes, die sich der langen Tradition kolonialen europäischen Verhaltens sehr bewusst war. In dieser Subgattung des Reiseberichtes haben wir es nicht mehr länger mit einem allmächtigen westlichen Subjekt zu tun, das sich alle Gegenstände aneignet und gleichsam einverleibt, sondern mit einer Vielfalt von Stimmen, deren Herkünfte wir nicht immer identifizieren können. Es handelt sich hier, um es pointiert zu formulieren, um einen Reisebericht ohne eigentlichen Bericht und vor allem ohne eine Reise, die innerhalb des Textes irgendwie greifbar würde. Dies stellt wiederum gänzlich andere Anforderungen an unser Analyseinstrumentarium. Aber darüber sprechen wir im historischen Teil unserer Vorlesung.

Die neunte Dimension des Reiseberichts ist über lange Jahrhunderte hinweg im Grunde (scheinbar) neutral gehalten worden. Nichts machte auf sie aufmerksam, denn die Frage schien sich generell nicht zu stellen. Die genderspezifische Dimension ist aber gerade dann, wenn sie als nicht pertinent dargestellt wird, sehr wohl auf impertinente Weise pertinent und gleichsam ubiquitär. Denn wenn Männer über Jahrhunderte hinweg ihr Reise-Schreiben so abfassten, dass ihre Reiseberichte genderspezifisch nicht ausgezeichnet oder markiert

23 Barthes, Roland: Sollers écrivain. Paris: Seuil 1979, S. 47. 
waren, dann bedeutet dies nicht, dass sie keine genderspezifische Position eingenommen hätten, sondern dass diese die im Grunde 'selbstverständliche', gleichsam 'natürliche' war, die in jeglicher Hinsicht vorherrschte. Nein: 'Natürlich' war dies aber ganz und gar nicht.

Frauen bildeten im literarischen Reisebericht als schreibende Subjekte die Ausnahme. Ihnen oblag es folglich, die eigenen Texte genderspezifisch zu markieren, um sie von der 'Normalität', nämlich dem männlichen Reisebericht, abzuheben. Dies aber hatte Folgen - und zwar auch für den Normalfall männlicher Texterzeugung. Wie ist dies zu verstehen?

In der Bewegung der Markierung des eigenen weiblichen Standpunktes wird gleichzeitig anschaulich, dass der 'Normalfall' des Reiseberichts sehr wohl genderspezifisch markiert war: Nur galten seine Codes nicht als Kodierungen, sondern als gleichsam 'natürliche' Norm und Normalität. Wir können aus der Differenz aber sehen, dass die genderspezifische Markierung sehr wohl gegeben und männlich beherrscht war. Frauen erschienen darin sehr wohl in ihrer Alterität als 'andere' Wesen: Sei es in ihrer radikalen Unterwerfung unter den Mann etwa in indigenen Gemeinschaften, sei es in der reizenden Atmosphäre schöner Salons, in denen sich die Männer von ihren Reisen erholten. Blickwechsel als Wechsel der Perspektive waren hier nur selten einmal anzutreffen: Der weibliche Blick auf das Reisen wie auf das Reisen-Können wurden nur selten signalisiert und (männlich) reflektiert. Frauenreisen nahmen erst im 19. Jahrhundert mehr und mehr zu, auch wenn sie immer noch bedeutsame Ausnahmen bildeten.

Auch wenn es zu allen Zeiten weibliche Reiseberichte gegeben hat, so werden sie erst im 19. Jahrhundert deutlich zahlreicher: Der Begriff der 'Frauenreise' etabliert sich. Kein Wunder aber, dass es den Begriff der 'Männerreise' schlicht nicht gibt. Frauen als Reiseschriftstellerinnen beziehen in ihren Texten - wie wir noch sehen werden - eine klare geschlechtliche Position, so wie es Flora Tristan in ihrem bereits erwähnten transatlantischen Reisebericht tat: Und sei es schon auf der Überfahrt die Tatsache, die einzige Frau an Bord gewesen zu sein und sich entsprechend verhalten zu müssen.

Die ebenfalls französische Schriftstellerin George Sand wiederum hat in ihrem Reisebericht, der unter dem Titel Un hiver à Majorque, der erstmals in der Revue des deux Mondes 1841 erschien, ihr Zusammenleben mit Frédéric Chopin geschildert und dabei eine klare genderspezifische Deutung ihrer Reiseerfahrung hinterlassen, wobei sie als Französin voller Abscheu davon berichtet, wie penetrant man sie im spanischen Mallorca oft von Frauenseite her an ihre spezifisch weiblichen Pflichten und Obliegenheiten erinnerte. In der Erfahrung einer anderskulturellen Differenz wird die eigenkulturelle Emanzipation mit ihren Schwierigkeiten neu beleuchtet, erscheint aber auch als Zeugnis einer Ungleich- oder Gleichbehandlung als Frau in Abhängigkeit von kulturellen Kontexten, die sich durch den Kontrast 
mit anderen Ländern deutlicher konturieren lassen. Die genderspezifische Markierung der eigenen (fortschrittlichen) Position als Französin war George Sand wie Flora Tristan eigen. Dies erinnert daran, dass wir nicht vergessen dürfen, uns mit dem allgemeinen kulturellen Raum gebührend auseinanderzusetzen und ihn in unsere Überlegungen miteinzubeziehen.

Wenden wir uns nun also der zehnten Dimension zu, die höchst komplex aufgebaut ist und der im Grunde eine übergeordnete Funktion zukommt. Denn sie wird vom kulturellen Raum gebildet, der in gewisser Weise die anderen Räume beziehungsweise Dimensionen quert. Raum und Zeit sind sehr wohl kulturell definiert und müssen folglich auch in dieser Hinsicht untersucht werden. Denn jede Kultur definiert eine ihr eigene Zeitlichkeit, in welche die beobachteten Phänomene eingeschrieben werden.

In Bezug auf die Reiseliteratur, aber gerade auch in Hinblick auf eine im weitesten Sinne grenzüberschreitende Literaturpraxis kommt der jeweiligen Positionierung eines Textes gegenüber bestimmten kulturellen Polen eine grundlegende Bedeutung zu. Die zehnte Dimension des kulturellen Raumes ist in jedem, selbst einem monokulturellen Text - gäbe es ihn denn - präsent, erhält aber gerade in der Reiseliteratur eine ungeheure Relevanz und Bedeutsamkeit mit Blick auf die Frage, wie anderskulturelle Erscheinungen jeweils literarisch, ästhetisch, aber auch politisch, gesellschaftlich oder philosophisch 'verarbeitet' und eingearbeitet werden. Dabei sind Relationen vorstellbar, die jenseits des Monokulturellen - sich auf ein multikulturelles Nebeneinander verschiedener, aber nicht miteinander in Kontakt stehender Kulturen, auf ein interkulturelles Miteinander zwischen verschiedenen Kulturen, die miteinander etwa in dialogischem Austausch stehen, oder auf ein transkulturelles Durcheinander beziehen, das in einem gänzlich positiven Sinne ein Queren und wechselseitiges Durchdringen verschiedenartiger kultureller Pole meint.

Die zehnte Dimension ist damit von fundamentaler Bedeutung für ein Verständnis des Reiseberichts bezüglich seiner kulturellen Koordinaten und Orientierungen. Darunter ist in der Folge ebenso die kulturelle Attribuierung bestimmter kultureller Bedingungen auf der Ebene der Objekte des Reiseberichts gemeint wie etwa die Markierung eigenkultureller Positionen beispielsweise auf der Ebene der reiseliterarischen Subjekte, mithin also die Bereitschaft, die eigenen Positionen im Lichte anderskultureller Gruppen, Gemeinschaften oder Verhaltensweisen zu sehen und zu erörtern. Wir werden uns die spezifischen Bedingungen und Relationen des kulturellen Raumes im Verlauf unseres historischen Analyseteiles noch genauer anschauen.

Denn die in diesem Kapitel vorgestellten und voneinander unterschiedenen Dimensionen sollen in der Folge flexibel aufgefasst und innerhalb jeweils unterschiedlicher Kontexte betrachtet und untersucht werden. Gewiss wäre es möglich, 
weitere Dimensionen des Reiseberichtes wie von Literatur überhaupt auszumachen und bei den analysierten Texten voneinander mehr oder minder klar zu unterscheiden. Vieles davon wird in den nachfolgenden Analysen anhand konkreter Beispiele entwickelt und vorgeführt werden. Zunächst jedoch gilt es, die vielfältigen und oftmals überraschenden Beziehungen zwischen Literatur und Reisen genauer herauszuarbeiten. 